\title{
Association of Health Risks With the Cost of Time Away From Work
}

\author{
Douglas W. Wright, PhD, \\ Marshall J. Beard, CEBS, CLU, ChFC \\ D. W. Edington, PhD
}

The purpose of this study was to combine absences, short-term disability, and workers' compensation into a sum of the cost of time away from work (TAW) and compare it with health risk status and individual health risks of 6220 hourly workers at Steelcase Inc. The study used 3 years (1998 to 2000) of TAW and health risk appraisal data. Higher TAW costs were associated with illness days, drug/ medication use, the individual's lower perception of physical health, job dissatisfaction, high stress, life dissatisfaction, and physical inactivity. More high-risk individuals (80.6\%) had a TAW occurrence than medium- $(72.8 \%)$ and low-risk (61.1\%) individuals. High-risk individuals had higher TAW costs than medium- and low-risk individuals. Of the total TAW costs, $36.2 \%$ was attributed to the excess risks of the medium- and high-risk individuals or nonparticipants compared with low-risk participants. If TAW costs follow risk reduction, a potential annual savings of $\$ 1.7$ million could be achieved. (J Occup Environ Med. 2002;44:1126-1134)
From the Health Management Research Center, University of Michigan, Ann Arbor (Dr Wright); Benefits and Health Services Steelcase, Inc., Grand Rapids, Michigan (Mr Beard); Health Management Research Center University of Michigan, Ann Arbor (Dr Edington).

Address correspondence to: D. W. Edington, PhD, Health Management Research Center, University of Michigan, 1027 E. Huron St., Ann Arbor, MI 48104-1688; e-mail: dwe@umich.edu Copyright $\odot$ by American College of Occupational and Environmental Medicine

DOI: 10.1097/01.jom.0000038330.36316.7e ime away from work has been looked at in a variety of ways depending on the data available. Time away from work (TAW) has generally involved scattered absences, short-term disability, and workers' compensation. Usually, each of these three measures has been studied separately, rather than in combination. This article examines all three measures and combines them into one total measure of the cost of overall TAW. The reason for combining all three measures is to give a broader view of the economic impact of overall TAW on a corporation.

The National Safety Council (NSC) estimated that on the job injuries cost the nation $\$ 131.2$ billion in the year 2000. ${ }^{1}$ This figure includes wage and productivity losses of $\$ 67.6$ billion, medical costs of $\$ 24.2$ billion, and administrative costs of $\$ 22.3$ billion. Employers spent another $\$ 11.5$ billion for time lost by employees who were not injured but involved in the reporting and investigation of injuries. As a result of the on the job injury cost, each worker must produce an extra $\$ 960$ in goods or services to offset the cost of work injuries. Three-fifths of all worker injuries actually occur off the job. It is estimated that offthe-job injuries to workers cost the nation at least $\$ 178.6$ billion in $2000 .^{1}$ In addition to the cost, offthe-job injuries accounted for $160,000,000$ days of lost production compared with $80,000,000$ days of lost production for on the job injuries in 2000. Although the on-the-job injury costs seem staggering, the $\mathrm{Na}$ tional Safety Council cost estimates 
may actually underestimate the true cost by $25 \%{ }^{2}$

Although the cost of on-the-job injuries keeps increasing, the rate of injury/illness involving days away from work has steadily declined from 3.0 per 100 workers in 1992 to 1.9 per 100 workers in 1999 . The total number of cases has also declined from 2.33 million in 1992 to 1.7 million in $1999 .^{3}$

\section{Association of Risk and Time Away From Work}

Although some of the on-the-job injury costs can be reduced through safety improvements and modified working environments, researchers have also shown an association between health risks and worker injury, illness, and absenteeism. Low exercise, ${ }^{4,5}$ depression, ${ }^{5,6}$ stress, ${ }^{7}$ being overweight or obese, ${ }^{5,8,9}$ smoking, 5,9 and low seat belt use ${ }^{5,9}$ have all been associated with increased absenteeism. Aldana and Pronk, ${ }^{10}$ Aldana, ${ }^{11}$ and Riedel et al ${ }^{12}$ have completed comprehensive reviews of the literature concerning the relationship between health risks and absenteeism. In all three reviews, being overweight/obese and high stress had the highest correlation with absenteeism.

Health risks have also been associated with decreased productivity. General distress, diabetes, and high BMI, ${ }^{13}$ as well as allergies, ${ }^{14,15}$ have been shown to be related to lowered productivity.

Other studies have linked pneumonia and disability claims, ${ }^{16}$ infrequent aerobic exercise, high work stress, and general worries with lower-back disability ${ }^{17,18}$ and substance abuse with time-loss injuries. ${ }^{19}$

Musich et $\mathrm{al}^{20}$ showed that high workers' compensation costs were related to health age index, smoking, poor physical health, physical inactivity, life dissatisfaction, and overall risk status.

\section{Association of Time Away From} Work and Health Promotion

In addition to showing the relationship between health risks and time away from work, there have been numerous studies on whether or not wellness programs have an effect on reducing health risks and as a result reducing the cost of worker absence. The evaluations of health promotion programs at the DuPont Company, ${ }^{21}$ Johnson \& Johnson, ${ }^{22}$ Duke University, ${ }^{23}$ General Mills, ${ }^{24}$ and Butterworth Hospital ${ }^{25}$ have all shown program participation to be associated with reduced absenteeism.

Participation in health-promotion programs also has been associated in separate studies with a $14 \%$ decline in disability days ${ }^{26} 6$ fewer days of short-term disability (STD), ${ }^{27}$ a 2.6 fold decrease in the number of lost workday injuries, ${ }^{28}$ and a savings of $\$ 1238$ in workers' compensation costs. $^{20}$

The purpose of this study was to combine hours of scattered absences, STD benefits, and workers' compensation (WC) costs into a measure of total time away from work (TAW) cost. We then examined the relationship of those TAW costs to health risk levels and individual health risks. We also examined the excess TAW cost of higher risk individuals and the relationship of HRA participation and TAW cost. Based on a review of the literature, we believe that this may be the first time anyone has combined scattered absences, STD, and WC into one overall TAW cost measure that was then associated with health status. The combination of all three measures into one cost should provide a new dimension in the areas of health promotion and work-loss benefits management.

\section{Methods}

Steelcase, Inc., based in Grand Rapids, MI, is a major manufacturer of office equipment and furniture. Steelcase initiated their wellness program (WELL, WELL, WELL!) in 1982, and it shares the company mission of "To help people work more effectively-no matter when, where or how they work." Because of the excellence of their wellness program, Steelcase won the prestigious C. Everett Koop National Health Award ${ }^{29}$ in 1994, which was the first year of the award. The health and costs of Steelcase employees have been continually tracked since 1985 , making it one of the longestrunning evaluated health promotion programs in the nation. Using Steelcase data, several studies have been published examining the relationships between health risks and medical claims, ${ }^{30}$ prediction of medical claims and absenteeism, ${ }^{31}$ medical cost distributions, ${ }^{32}$ and the financial impact of changing health behavior. ${ }^{33}$ The major conclusions of these four studies are that medical costs and absenteeism increase as health risks increase and that a change in risk behavior results in a decrease in costs.

\section{Study Population}

This study examined relationships between health risks, health status, and TAW costs. To be included in the study, employees had to be employed at Steelcase for the full 3 years of the study (1998 to 2000) and be an hourly employee. A total of 6220 active hourly employees met the inclusion criteria. WC, STD, and scattered absence from work (ABS), were combined to give an overall TAW cost during the time period for each individual. Throughout this article, the term "time away from work (TAW)" or "time away from work cost (TAW)" will refer to the sum of WC, STD, and ABS on an average annual basis. WC, STD, and calculated ABS costs from 1998 to 2000 were used. Employees with no TAW occurrence in the 3-year time period were given a value of zero. Employees could belong to any of the health care plans available (HMOs, traditional/PPO) because plan choice should have minimal effect on TAW costs.

\section{Absence Cost Calculations}

The absence (ABS) data available from Steelcase contained hours of absence on a daily basis for all ab- 
sent hourly employees. Absence records included both sickness, and approved and unapproved medical leave. Individual summed absence records, within a year, resulted in the total hours of absence per individual for each year. Individual wages were unavailable; however, because of the type of manufacturing and length of employment necessary to be in the study, the wage range was relatively narrow. Therefore, absence hours were converted to ABS cost by multiplying the number of hours by $\$ 25$. The $\$ 25$ per hour ( $\$ 200 /$ day for a standard 8-hour work day) amount represents the estimated direct and indirect cost for hourly wages at a manufacturing company. Others have shown daily values ranging from $\$ 150^{20}$ to $\$ 225$. $^{27}$

\section{STD Claims}

At Steelcase, STD benefits start after an employee has 4 consecutive absence days for sickness or disease, or 1 day after an accident. STD can last a maximum of 6 months, at which point it then becomes a longterm disability. Employees with STD receive a set amount of $\$ 350$ per week from the employer and after 2 weeks of STD, they may receive an additional amount of benefits if the employee paid extra for STD insurance. The additional amount can vary from $\$ 50$ to 150 per week. The data available to us from a thirdparty payer gave the total monetary benefit for each STD incident, combining both the employer benefit and the supplemental insurance benefit (if any) into one value. STD costs were calculated by summing claims, with a beginning claim date within the specified year, for each individual. Because the duration of the STD claims was not available, some STD claims may actually span multiple years but were only accounted for in the beginning claim year. However, because the outcome is average annual costs, the effect should be minimal.

Some of the employees with STD also appeared in the ABS data for the same time period. To minimize double counting, if a STD and ABS occurred at the same time, the ABS hours were reduced by taking the STD cost and dividing by $\$ 50$ (the company STD benefit per day) and then subtracting the resulting number of hours ( 8 hours per day) from the ABS claim. Because the employee may get more STD money than the company benefit, we erred on the conservative side and used the smaller amount per day to subtract the greatest possible number of days from the ABS data.

\section{WC Claims}

The WC data available to us from a third-party payer included medical costs, wage compensation costs, internal and external attorney's fees, and miscellaneous fees for each individual per incident. Summing those costs gave the total WC cost per individual per incident. Similar to the STD data, WC claims did not have a length of duration, so some WC costs may actually span multiple years but were only accounted for in the beginning claim year.

\section{Health Risk Appraisal}

The first health risk appraisal (HRA) at Steelcase was offered in 1985 and given on a 3-year rotating basis to completely cover all departments. In 1993, the HRA rotation was shortened to 2 years to cover all departments. Starting in 1998, Steelcase began offering the HRA to all employees on an annual basis. All HRAs include a biometric screening with health counseling provided by either a third party or by a case manager if they belong to an HMO. In addition to the HRA biometric screening, employees have access to various wellness programs, newsletters, and healthy meal choices in the cafeteria.

Fifteen health-related factors measured on the HRA were selected to establish health status. A list of the risk factors and their associated highrisk criteria is shown in Table 1 . The risks fall into three main categories: lifestyle/biological, psychological, and other. Lifestyle/biological risks include smoking, low physical activity, high alcohol use, drug use (including prescription) that affects mood or helps one to relax, less than $100 \%$ use of safety belts, absences of 6 days or longer because of illness, medical problems (heart, cancer, diabetes, stroke, or bronchitis/emphysema), high blood pressure, cholesterol greater than 239, and body weight $20 \%$ over desirable. Psychological risks include fair or poor perception of health, partly or not satisfied with personal life, low job satisfaction, and a stress score over 18. One additional risk included was health age index, where the appraised age minus the achievable age (from HRA algorithms) is greater than 4 years.

Health risks and health status are based on the earliest of the 1998, 1999, and 2000 HRAs (each with 15 possible risks). The total number of participants was 696 for the 1998 HRA, 806 for the 1999 HRA, and 746 for the 2000 HRA. A total of 1571 employees (25\% of study population) took at least one HRA.

Risk levels were defined as 0 to 2 risks being low-risk, 3 to 4 risks being medium-risk, and 5 or greater risks being high-risk, based on a maximum of 15 risks.

\section{Statistical Testing}

All statistical procedures were performed using SAS. ${ }^{34}$ Categorical variables were tested using the Pearson chi-square statistic. Because annual TAW costs were highly skewed (a mean of $\$ 768$ and a median of $\$ 138$ for all employees), a $\log 10$ $(\operatorname{cost}+1)$ transformation was used before mean comparisons. TAW costs were analyzed using the General Linear Model procedure in SAS, adjusting for the main effects of gender and age group (19 to 34,35 to 44 , 45 to 54 , and 55 to 64 years old). Least-squares means were computed and compared using the PDIFF option with a Tukey-Kramer adjustment for multiple comparisons. 


\section{TABLE 1}

High Health-Risk Criteria

\section{Selected Measures}

\begin{tabular}{|c|c|}
\hline \multicolumn{2}{|l|}{ Lifestyle/biological risks } \\
\hline Smoking & Current cigarette smoker \\
\hline Physical activity & $<1$ time per week \\
\hline Alcohol use & Heavy drinker (>14 drinks/week) \\
\hline Drug/medication use* & Sometimes or almost every day \\
\hline Safety belt use & Using safety belt less than $100 \%$ of the time \\
\hline Absent because of illness & Six days or more during last year \\
\hline Medical problems & $\begin{array}{l}\text { Had problems with heart condition, cancer, diabetes, } \\
\text { stroke, or bronchitis/emphysema }\end{array}$ \\
\hline Blood pressure & $\begin{array}{l}\text { Systolic blood pressure greater than } 139 \mathrm{~mm} \mathrm{Hg} \text { or dia- } \\
\text { stolic blood pressure greater than } 89 \mathrm{~mm} \mathrm{Hg} \text { or taking } \\
\text { blood pressure medication or self reported high }\end{array}$ \\
\hline Cholesterol & Greater than $239 \mathrm{mg} / \mathrm{dL}$ \\
\hline Body weight & Over desirable weight $20 \%$ or more \\
\hline \multicolumn{2}{|l|}{ Psychological risks } \\
\hline Personal life satisfaction & Partly satisfied or not satisfied \\
\hline Job satisfaction & Satisfied with job? (disagree or disagree strongly) \\
\hline Stress & Stress-scale score over 18 \\
\hline Perception of health & Fair or poor \\
\hline \multicolumn{2}{|l|}{ Other risk } \\
\hline Health Age Index & Appraised age minus achievable age (from HRA) $>4$ years \\
\hline \multicolumn{2}{|l|}{ Overall risk levels } \\
\hline Low risk & $0-2$ high risks \\
\hline Medium risk & 3-4 high risks \\
\hline High risk & 5 or more high risks \\
\hline
\end{tabular}

* Use of medication and/or drugs (including prescription), that affect mood or help to relax.

\section{TABLE 2}

\begin{tabular}{|c|c|c|}
\hline Demographics & $\begin{array}{c}\text { With TAW } \\
\text { Occurrences } 1998 \text { to } 2000 \\
(n=4090)\end{array}$ & $\begin{array}{c}\text { Without TAW } \\
\text { Occurrences } 1998 \text { to } 2000 \\
(n=2130)\end{array}$ \\
\hline \multicolumn{3}{|l|}{ Gender* } \\
\hline Male $(n=5315)$ & 3363 (82.2\%) & 1952 (91.6\%) \\
\hline Female $(n=905)$ & 727 (17.8\%) & $178(8.4 \%)$ \\
\hline \multicolumn{3}{|l|}{ Age, years* } \\
\hline $19-34(n=1071)$ & 799 (19.5\%) & $272(12.8 \%)$ \\
\hline $35-44(n=2516)$ & 1631 (39.9\%) & $885(41.5 \%)$ \\
\hline $45-54(n=2084)$ & $1338(32.7 \%)$ & 746 (35.0\%) \\
\hline $55-64(n=549)$ & $322(7.9 \%)$ & 227 (10.7\%) \\
\hline \multicolumn{3}{|l|}{ HRA Participant* } \\
\hline Yes $(n=1571)$ & $1096(26.8 \%)$ & 475 (22.3\%) \\
\hline No $(n=4649)$ & 2994 (73.2\%) & 1655 (77.7\%) \\
\hline
\end{tabular}

${ }^{*} P<0.01$, chi-square.

\section{Results}

Demographic characteristics of the employee population by TAW status are shown in Table 2. There were differences $(P<0.01)$ in the number of people with TAW occurrences for gender, age class, and HRA participation. There were a higher percentage of females among those with TAW than those without TAW. A higher percentage of young workers
(19-34) had a TAW occurrence compared with those without a TAW occurrence. HRA participants were more likely to have a TAW occurrence than the nonparticipants.

The TAW and component (ABS, STD, WC) costs by year are shown in Table 3. Total TAW costs increased from 1998 to 2000, although the increases were minimal from 1998 to 1999 . From 1999 to 2000 ,
TAW costs increased by $15.7 \%$ from $\$ 4,552,265$ to $\$ 5,269,624$, even though the number of employees with an occurrence decreased from 2782 to 2713 . The percentage of employees with a TAW occurrence has remained in a narrow range with a low of $41.0 \%$ in 1998 and a high of $44.7 \%$ in 1999. Mean TAW costs were lowest in 1999 (\$1636) and increased to $\$ 1942$ per employee with an occurrence in 2000. Overall, $65.8 \%$ of the hourly employees had a TAW occurrence during the threeyear period accounting for just over $\$ 14.3$ million in TAW costs.

ABS costs doubled from 1998 to 1999, and the number of employees with an absence increased by $20 \%$. Total ABS costs decreased from 1999 to 2000, as did the number of employees with an absence. STD and WC costs have gone up and down, with a low in 1999. At the same time, the number of employees with an STD peaked in 1999 whereas the number of employees with a WC claim was at a minimum. The costs of STD and WC increased from 1999 to 2000 , creating the $15.7 \%$ increase in overall TAW costs mentioned previously. For WC, both the number of employees with a claim and the cost increased from 1999 to 2000 . Overall, WC accounted for $45.3 \%$ of the TAW costs, followed by ABS $(31.8 \%)$, and STD (22.9\%).

Average costs for TAW and the three TAW components by risk level and HRA participation are shown in Table 4. The costs are only for those with an occurrence in their respective categories. The high-risk group of employees had a greater mean annual TAW cost $(\$ 1764)$ than the medium-risk $(\$ 1224, P<0.01)$ and low-risk groups $(\$ 1096, p<0.01)$. The medium-risk group TAW cost was greater than the low-risk group but was not significantly different.

The high-risk group had the greatest ABS cost (\$764), followed by the medium-risk (\$541) and low-risk (\$493) groups, with the low-risk group being different from the high-risk group $(P<0.01)$. For STD, the high-risk 
TABLE 3

TAW and TAW Component Costs With Number of Employee* Occurrences by Year

\begin{tabular}{|c|c|c|c|c|c|c|c|c|}
\hline \multirow[b]{2}{*}{ Year } & \multicolumn{2}{|c|}{ TAW } & \multicolumn{2}{|r|}{ ABS } & \multicolumn{2}{|r|}{ STD } & \multicolumn{2}{|r|}{ WC } \\
\hline & Employees & Cost $^{\dagger}$ & Employees & Cost ( $\%$ of Total) & Employees & Cost (\% of Total) & Employees & Cost (\% of Total) \\
\hline 1998 & 2552 & $\$ 4,511,898$ & 1784 & $\$ 975,089(21.6 \%)$ & 851 & $\$ 1,094,699(24.3 \%)$ & 838 & $\$ 2,442,110(54.1 \%)$ \\
\hline 1999 & 2782 & $\$ 4,552,265$ & 2140 & $\$ 1,843,783(40.5 \%)$ & 924 & $\$ 870,284(19.1 \%)$ & 743 & $\$ 1,838,198(40.4 \%)$ \\
\hline 2000 & 2713 & $\$ 5,269,624$ & 2076 & $\$ 1,747,854(33.2 \%)$ & 875 & $\$ 1,312,465$ (24.9\%) & 777 & $\$ 2,209,305(42.9 \%)$ \\
\hline Total & 4090 & $\$ 14,333,786$ & 2830 & $\$ 4,566,725$ (31.8\%) & 1865 & $\$ 3,277,448$ (22.9\%) & 1875 & $\$ 6,489,613(45.3 \%)$ \\
\hline
\end{tabular}

${ }^{*}$ Number of employees each year with an occurrence within a category.

† Sum of ABS, STD, and WC costs.

\section{TABLE 4}

Mean Annual TAW Costs* by Risk Level and HRA Participation Among Those With Occurrences

\begin{tabular}{|c|c|c|c|c|}
\hline Risk Level & Mean TAW Cost ${ }^{\star \star}$ & Mean ABS Cost ${ }^{\star *}$ & $\begin{array}{l}\text { Mean STD } \\
\text { Cost }^{\star \star}\end{array}$ & Mean WC Cost ${ }^{\star *}$ \\
\hline Med risk $(n=504)$ & $\$ 1,224^{\mathrm{b}}(n=367)$ & $\$ 541^{\mathrm{abd}}(n=318)$ & $\$ 694^{\mathrm{c}}(n=157)$ & $\$ 826^{c}(n=149)$ \\
\hline HRA participant $(n=1,571)$ & $\$ 1,243^{\mathrm{c}}(n=1,096)$ & $\$ 590^{\mathrm{a}}(n=925)$ & $\$ 653^{c}(n=491)$ & $\$ 900^{\circ}(n=470)$ \\
\hline HRA nonparticipant $(n=4,649)$ & $\$ 1,416^{\mathrm{c}}(n=2,994)$ & $\$ 686^{\mathrm{b}}(n=1,905)$ & $\$ 714^{\mathrm{d}}(n=1,374)$ & $\$ 1,218^{\mathrm{c}}(n=1,405)$ \\
\hline
\end{tabular}

* Means adjusted for gender and age category.

${ }^{* *}$ Employees with an occurrence within a category.

a,b Means in a column with differing letters, within risk level or HRA participation, are statistically significant at $P<0.01$ ANOVA (log 10 $(\cos t+1))$.

${ }_{\mathrm{c}, \mathrm{d}}$ Means in a column with differing letters, within risk level or HRA participation, are statistically significant at $P<0.05$ ANOVA (log 10 $(\cos t+1))$.

\section{TABLE 5}

Percentage of Low-, Medium-, and High-Risk Participants and Nonparticipants With a TAW Occurrence (1998 to 2000)

\begin{tabular}{llllr}
\multicolumn{1}{c}{ Risk Level } & \multicolumn{1}{c}{ All TAW } & \multicolumn{1}{c}{ ABS } & \multicolumn{1}{c}{ STD } & WC \\
\hline High risk $(n=396)$ & $80.6 \%^{\mathrm{a}}(n=319)$ & $68.9 \%^{\mathrm{a}}(n=273)$ & $45.2 \%^{\mathrm{a}}(n=179)$ & $37.6 \%^{\mathrm{ad}}(n=149)$ \\
Med risk $(n=504)$ & $72.8 \%^{\mathrm{b}}(n=367)$ & $63.1 \%^{\mathrm{a}}(n=318)$ & $31.2 \%^{\mathrm{b}}(n=157)$ & $29.6 \%^{\mathrm{e}}(n=149)$ \\
Low risk $(n=671)$ & $61.1 \%^{\mathrm{c}}(n=410)$ & $49.8 \%^{\mathrm{b}}(n=334)$ & $23.1 \%^{\mathrm{c}}(n=155)$ & $25.6 \% \%^{\mathrm{be}}(n=172)$ \\
HRA participant $(n=1,571)$ & $69.8 \%^{\mathrm{a}}(n=1,096)$ & $58.9 \%^{\mathrm{a}}(n=925)$ & $31.3 \%^{\mathrm{d}}(n=491)$ & $29.9 \%^{\mathrm{d}}(n=470)$ \\
HRA nonparticipant $(n=4,649)$ & $64.4 \%^{\mathrm{b}}(n=2,994)$ & $41.0 \%^{\mathrm{b}}(n=1,905)$ & $29.6 \%^{\mathrm{d}}(n=1,374)$ & $30.2 \%^{\mathrm{d}}(n=1,405)$ \\
\hline
\end{tabular}

a,b,c $P<0.01$, chi-square, within a column and within risk level and HRA participation.

${ }^{\mathrm{d}, \mathrm{e}} P<0.05$, chi-square, within a column and within risk level and HRA participation.

group had the highest cost (\$737) followed by the medium-risk group (\$694), and the low-risk group (\$521), although none of the differences were significant. The WC costs were unusual in that the medium-risk group had the lowest cost, whereas in the other TAW categories, the low-risk group had the lowest costs. The highrisk group had greater WC costs (\$1318) than the low-risk (\$890) and medium-risk (\$826) groups. There were no significant differences in WC cost between the three risk groups.

HRA participants had lower annual TAW ( $\$ 1243, P=0.31)$, ABS
(\$590, $P<0.01)$, STD $(\$ 653, P<$ $0.05)$, and WC $(\$ 900, P=0.85)$ costs than the HRA nonparticipants (\$1416, \$686, \$714, and \$1218, respectively).

The percentages of employees within each risk level that had a TAW occurrence are shown in Table 5. All three risk level groups were different $(P<0.05)$ from each other for all four categories of TAW, except for the not significant WC difference between the medium- and low-risk groups and the non-significant ABS difference between the medium and high-risk groups. The risk level groups showed the same trend in each of the four TAW categories, where the high-risk group had the highest percentage followed by the medium- and low-risk groups. For total TAW, $80.6 \%$ of the high-risk employees had an occurrence followed by medium risk $(72.8 \%)$ and low risk (61.1\%). For ABS, STD, and WC, the high-risk group had $68.9 \%, 45.2 \%$, and $37.6 \%$; the medium-risk group had $63.1 \%, 31.2 \%$, and $29.6 \%$; and the low-risk group had $49.8 \%, 23.1 \%$, and $25.6 \%$ of occurrences, respectively. 


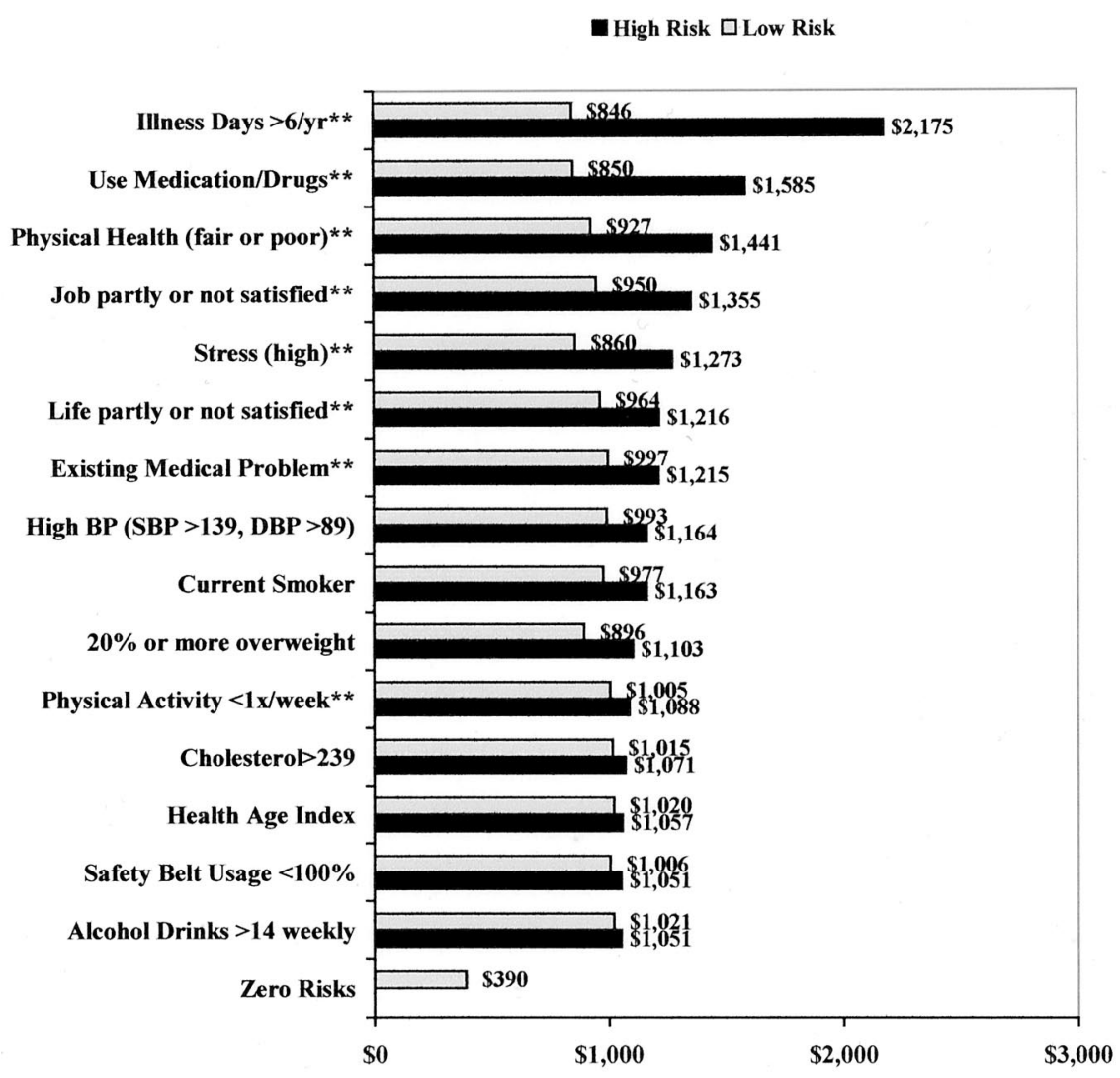

$* \mathrm{P}<0.05$ ANOVA $(\log 10(\cos t+1))$. Means adjusted for gender and age category. $* * \mathrm{P}<0.01$ ANOVA $(\log 10(\cos t+1))$. Means adjusted for gender and age category.

Fig. 1. Mean annual TAW cost by risk status ( $n=1571$ with HRA). $* P<0.05$ analysis of variance $(\log 10(\cos t+1))$. Means adjusted for gender and age category. $* * P<0.01$ analysis of variance $(\log 10(\operatorname{cost}+1))$. Means adjusted for gender and age category.

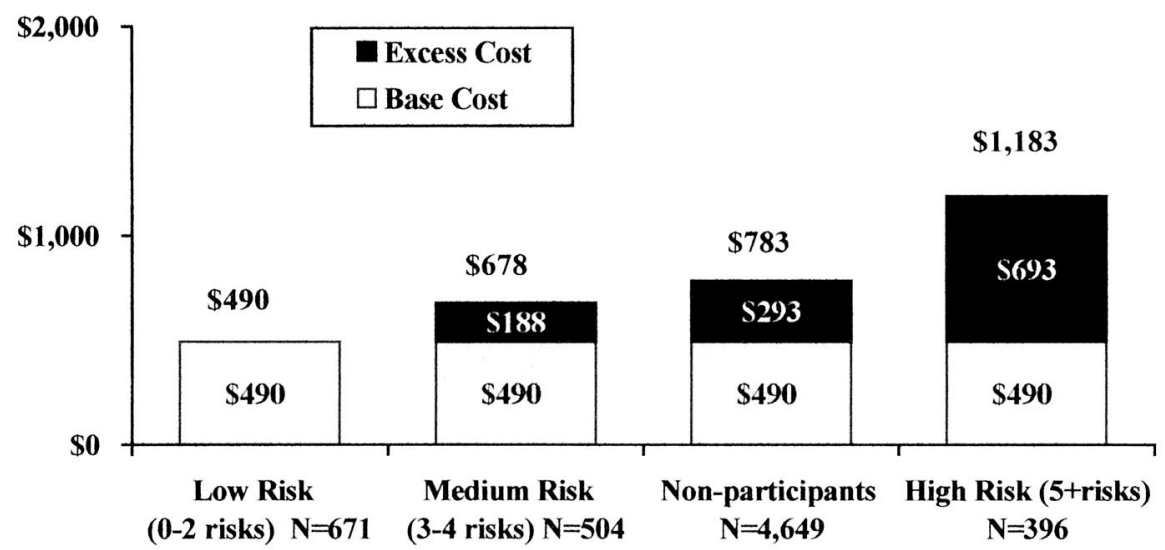

Fig. 2. Excess TAW costs related to excess risk

HRA participants had a higher percentage of TAW occurrences $(P<0.01)$, absence occurrence $(P<$ 0.01 ), and STD claims (NS), but had a lower percentage of WC claims (NS) than HRA nonparticipants.
Individual health risk status by TAW cost is shown in Fig. 1. Employees at high risk for illness days $>=6$ per year, fair or poor physical health, use of medication/ drugs to relax or alter mood, job dissatisfaction, high stress, existing medical problems, partly or not satisfied with life, or physical activity $<1$ time per week, had higher $(P<$ $0.01)$ TAW costs than employees without those individual risks. As might be expected, the cost differential was greatest for illness days $>=$ 6 per year $(\$ 2175$ for high risk, $\$ 846$ for low risk). Similar risk-cost trends were seen for the components of TAW (ABS, STD, WC, data not shown).

Excess TAW costs associated with excess risk are shown in Fig. 2. Medium-risk employees had $\$ 188$, high-risk had \$693, and HRA nonparticipants had \$293, in annual excess costs above the low risk base line of $\$ 490$. For HRA participants, $32.4 \%$ or $\$ 369,180$ in TAW costs is associated with excess risk. This is the theoretical maximum savings in annual TAW costs that could be achieved if all HRA participants changed to low risk and TAW costs followed this change in risk. If you include the excess TAW cost of HRA nonparticipants, which is $36.2 \%$ or $\$ 1731,337$, of the total annual TAW costs could be saved, assuming nonparticipants had a similar risk distribution as HRA participants and TAW cost followed the risk reduction to low risk.

\section{Discussion}

The strong association between health risk level and TAW costs is clear. Among those with a TAW occurrence, high-risk individuals had mean annual TAW costs $\$ 540$ and $\$ 668$ higher than medium- and lowrisk individuals, respectively (Table 4). High-risk individuals also had higher ABS, STD, and WC costs than the low-risk individuals. Almost $81 \%$ of high-risk individuals had a TAW occurrence during the 3-year period compared with $72.8 \%$ and $61.1 \%$ of medium- and low-risk employees, respectively. This same trend was seen for ABS, STD, and WC.

Individual health risks were also associated with TAW costs. Employ- 
ees at high risk for illness days, perception of physical health, use of medication/drugs to relax or alter mood, job dissatisfaction, high stress, existing medical problems, life dissatisfaction or lack of physical activity, had significantly higher TAW costs than employees without those individual risks. From the literature, individual risks associated with the components of TAW include exercise frequency, $, 5,17,20$ stress, ${ }^{5,7,13,17}$ weight, $, 5,8,9,13$ mental health, ${ }^{5,6}$ smoking, ${ }^{5,9,20,31}$ allergies, ${ }^{15}$ diabetes,${ }^{13}$ alcohol,${ }^{9}$ cholesterol, ${ }^{9,31}$ blood pressure,,${ }^{9,31}$ seat belt use, ${ }^{5,9}$ substance abuse, ${ }^{19,31}$ poor physical health, ${ }^{20}$ and life dissatisfaction. ${ }^{20}$

In this study, HRA participants had lower TAW, ABS, STD, and WC costs than nonparticipants, with the differences in ABS and STD reaching significance. However, HRA participants had a higher percentage of TAW, ABS, and STD occurrences than nonparticipants, with the differences in TAW and ABS reaching significance. This would indicate that even though participants are more likely to have a claim, the severity of the claim is less than that of a nonparticipant. HRA participants could have participated in other parts of the Steelcase wellness program that could influence costs, but even by taking just a HRA, it means that at a minimum they were given a biometric screening and counseling. Bertera ${ }^{26}$ showed a $14 \%$ decline in disability days for program participants and, similarly, this study showed that HRA participants had $13.9 \%$ lower TAW costs than nonparticipants.

Even though the purpose of this study was to look at the overall TAW costs, individual TAW component costs were calculated to show the influence of each component. WC accounted for $45.3 \%$ of the TAW costs, followed by ABS (31.8\%) and STD $(22.9 \%)$. Although WC costs made up a substantial portion of the TAW cost, it is self-evident that ABS and STD also contribute a significant amount to the overall TAW cost.

If the difference in annual ABS costs between HRA participants and nonparticipants with an ABS claim (\$96) is converted into time, then HRA participants had 3.8 fewer hours of annual absence than the nonparticipants. This is less than the difference of 4.6 hours of absence per year reported for the Duke University health promotion program, ${ }^{23}$ but more than the 0.15 annual absence days (1.2 hours/year) difference reported for hospital workers. ${ }^{25}$ However, those two studies compared the entire population, not just those with an absence. Hourly workers who participated in the health promotion program at Johnson \& Johnson showed a significant decrease in absence hours, ${ }^{22}$ as did the participants at General Mills ${ }^{24}$; however, the difference at General Mills came from nonparticipants increasing over time whereas the participants stayed the same. High-risk individuals at DuPont showed a $12 \%$ decline in absence days after 2 years of a health-promotion program but no difference for low-risk people. ${ }^{21}$

If the difference in STD costs between HRA participants and nonparticipants with a STD claim (\$61) is converted into time (at $\$ 50 /$ day), then HRA participants had 1.22 fewer days of STD annually compared with nonparticipants. Sexner et $\mathrm{al}^{27}$ looking at only those with a STD claim, found that participants in a work site health promotion program had 6 fewer days of STD than nonparticipants over a 2-year period (3.0 days/year), representing a $20 \%$ program impact, compared with the 9.3\% less STD cost in this study.

It is difficult to compare $\mathrm{WC}$ costs with other studies because of differences in state laws, medical costs, and wages. We were also limited by only having WC claims costs and not duration, but Musich et $\mathrm{al}^{20}$ found that HRA participants had \$444 (adjusted to an annual basis) less in WC claims cost compared with nonparticipants with claims, which com- pares favorably with the $\$ 318$ difference shown in this study.

In 1997, Steelcase integrated workers' compensation and shortand long-term disability into one department and showed an overall drop in costs from $\$ 1.55$ per $\$ 100$ of payroll in 1997 to $\$ 1.40$ in $1999 .{ }^{35}$ Those results are evident in this study, because STD and WC costs declined by $20.5 \%$ and $24.7 \%$, respectively, from 1998 to 1999 . However, those same costs increased by $50.8 \%$ and $20.2 \%$, respectively, from 1999 to 2000 , resulting in a $15.7 \%$ increase in total TAW costs. This would indicate that Steelcase has realized most of the benefits of integration and now must look to other sources to minimize or reverse the impact of the increase in TAW costs.

The relative closeness of the two excess cost percentages $(32.4 \%$ and $36.2 \%$ for HRA only and HRA plus nonparticipants, respectively) would indicate that the nonparticipant population is likely to have a similar distribution of health risk status levels if they were to take an HRA. The TAW costs of low risk employees were used as the baseline TAW cost, and any costs above that level in the other risk groups were considered to be caused by the elevated risk level. The potential annual cost savings of risk reduction was $\$ 369,180$ for HRA participants and $\$ 1,731,337$ if nonparticipants are included, assuming that TAW costs followed the change to low risk. Musich et $\mathrm{al}^{20}$ showed an excess cost of $85 \%$ for WC at Xerox; however, those results were for long-term employees (service of 18 years or longer) and included salaried workers. Looking at medical costs, Edington ${ }^{36}$ showed an excess cost of $21 \%$ to $31 \%$ with a mean of $25 \%$ for various corporate clients. This would imply that increased risk level affects TAW costs even more than medical costs.

TAW costs for the entire population were highly skewed with the top ten percent of the population accounting for $65 \%$ of the TAW costs (data not shown). The $\log 10(\mathrm{x}+1)$ 
transformation appeared to greatly lessen the skewness (data not shown), giving more confidence that the statistical comparisons are valid. The distribution of TAW costs (untransformed) was very similar to the distribution of WC costs shown by Musich et al. ${ }^{20}$

Overall, Steelcase TAW, ABS, STD, and WC costs are low compared with the national average and manufacturing industry in general. The national average WC cost per claim is $\$ 10,610$, and in Michigan, the average WC cost per case is $\$ 26,539,{ }^{3}$ compared with the $\$ 3461$ per person, with a WC claim, paid by Steelcase over 3 years. Steelcase also paid an average of $\$ 176$ in STD per worker per year over the 3-year period, compared with the $\$ 461$ paid by International Truck and Engine Corporation in $1999 .{ }^{37}$ Goetzel et $\mathrm{al}^{38}$ estimated that the median 1998 cost per eligible employee for ABS, STD, and $\mathrm{WC}$ was $\$ 810, \$ 513$, and $\$ 310$, respectively, in a survey conducted among 43 employers. In comparison, the $\$ 245, \$ 176$, and $\$ 348$ average per year for ABS, STD, and WC, respectively, indicates that Steelcase pays about $30 \%$ of the survey average for ABS and STD while paying slightly more for WC. Surprisingly, in a previous study of Steelcase hourly workers, Yen et $\mathrm{al}^{31}$ showed an average annual absence cost of $\$ 825$ per employee in 1986 dollars. This would indicate that Steelcase has been successful in reducing absenteeism over time, either through policy, health promotion, or both.

\section{Limitations}

This study examined the experiences of hourly workers who had been fully employed at Steelcase from 1998 to 2000. These results may not apply to the rest of the Steelcase workforce, although a high proportion of the hourly employees are long-term employees. Salaried workers have fewer chances to suffer major injuries on the job compared with hourly manufacturing employees, so their results may differ from the study population. However, salaried workers can still be injured off the job, as well as have scattered absences.

Absence costs were calculated from hours of absence using an estimate of $\$ 25 /$ hour in direct and indirect costs. Obviously if that is not the true value, then the overall cost estimates may be either over or under estimated. However, from personal communication with corporate benefits personnel it would appear that, if anything, the $\$ 25 /$ hour estimate was conservative.

The length of time was unknown for STD and WC claims, meaning that some costs included in the study could have actually been incurred after the study. However, since some costs attributed to 1997 may have actually occurred in 1998, the effects of not knowing the time period should be minimal.

Because WC costs include wage compensation, some WC costs may have been caused strictly by the earnings of the employee; however, the range of wages within the hourly population should be relatively minor compared with the differences in a salaried population. Having to be employed for a minimum of 3 years should also lessen the possible wage difference.

\section{Implications}

The association of TAW costs with health risk level and individual health risks was readily apparent. If a decrease in TAW costs follows a reduction in health risks, then the potential TAW cost savings could amount to $32 \%$ to $36 \%$ of total cost incurred, not to mention the potential medical savings shown in the literature. The over $\$ 1.7$ million in annual excess costs shown in this study, should be an impetus to continue or increase the funding of the current wellness program at Steelcase to promote a strategy for risk reduction and low risk maintenance, as well as provide positive evidence to other companies of the value of health promotion. Although traditional
TAW cost containment programs have also shown positive results, corporations can no longer ignore the link between health status and TAW costs.

This article combined ABS, STD, and WC claims into a measure of total TAW cost. By being able to assess the total impact of TAW on the corporation, health promotion professionals and benefits managers can more wisely allocate resources to benefit the employees.

\section{References}

1. National Safety Council. Injury Facts, 2001 Edition. Itaska, IL: NSC; 2001.

2. Leigh JP, Markowitz SB, Fahs M, Shin $\mathrm{C}$, Landrigan PJ. Occupational injury and illness in the United States: estimates of costs, morbidity, and Mortality. Arch Intern Med. 1997;157:1557-1568.

3. Bureau of Labor Statistics. Nonfatal cases involving days away from work. Available at: http://stats. bls. gov/iif.

4. Jacobsen BH, Aldana SG. Relationship between frequency of aerobic activity and illness-related absenteeism in a large employee sample. J Occup Environ Med. 2001;43:1019-1025.

5. Serxner SA, Gold DB, Bultman KK. The impact of behavorial health risks on worker absenteeism. J Occup Environ Med. 2001;43:347-354.

6. Claxton AJ, Chawla AJ, Kennedy S. Absenteeism among employees treated for depression. J Occup Environ Med. 1999;41:605-611.

7. Jacobson BH, Aldana SG, Goetzel RZ, Vardell KD, Adams TB, Pietras RJ. The relationship between perceived stress and self-reported illness-related absenteeism. Am J Health Promot. 1996;11:54-61.

8. Tucker LA, Friedman GM. Obesity and absenteeism: an epidemiologic study of 10,825 employed adults. Am J Health Promot. 1998;12:202-207.

9. Bertera RL. The effects of behavioral risks on absenteeism and health-care costs in the workplace. J Occup Med. 1991;33:1119-1124.

10. Aldana SG, Pronk NP. Health promotion programs, modifiable health risks, and employee absenteeism. J Occup Environ Med. 2001;43:36-46.

11. Aldana SG. Financial impact of health promotion programs: comprehensive review of the literature. Am J Health Promot. 2001;15:296-319.

12. Riedel JE, Lynch W, Baase C, Hymel P, Peterson KW. The effect of disease pre- 
vention and health promotion on workplace productivity: a literature review. Am J Health Promot. 2001;15:167-191.

13. Burton WN, Conti DJ, Chen CY, Schultz $\mathrm{AB}$, Edington DW. The role of health risk factors and disease on worker productivity. J Occup Environ Med. 1999; 41:863-877.

14. Cockburn IM, Bailit HL, Berndt ER, Finkelstein SN. Loss of work productivity due to illness and medical treatment. J Occup Environ Med. 1999;41:948-953.

15. Burton WN, Conti DJ, Chen CY, Schultz $\mathrm{AB}$, Edington DW. The impact of allergies and allergy treatment on worker productivity. J Occup Environ Med. 2001;43:64-71.

16. Birnbaum HG, Morley M, Greenberg PA, Cifaldi M, Colice GL. Economic burden of pneumonia in an employed population. Arch Intern Med. 2001;161: 2725-2731.

17. Feuerstein M, Berkowitz SM, Huang GD. Predictors of occupational low back disability: implications for secondary prevention. J Occup Environ Med. 1999; 41:1024-1031.

18. Feuerstein M, Berkowitz SM, Haufler AJ, Lopez MS, Huang GD. Working with low back pain: workplace and individual psychosocial determinants of limited duty and lost time. Am J Indust Med. 2001;40:627-638.

19. Pollack ES, Franklin GM, Fulton-Kehoe D, Chowdhury R. Risk of job-related injury among construction laborers with a diagnosis of substance abuse. J Oсcиp Environ Med. 1998;40:573-577.

20. Musich S, Napier D, Edington DW. The association of health risks with workers' compensation costs. J Occup Environ Med. 2001;43:534-541.
21. Bertera RL. Behavioral risk factor and illness day changes with workplace health promotion: two-year results. Am J Health Promot. 1993;7:365-373.

22. Jones RC, Bly JL, Richardson JE. A study of a work site health promotion program and absenteeism. J Occup Med. 1990;32:95-99.

23. Knight KK, Goetzel RZ, Fielding JE, et al. An evaluation of Duke University's LIVE FOR LIFE health promotion program on changes in worker absenteeism. J Occup Med. 1994;36:533-536.

24. Wood EA, Olmstead GW, Craig JL. An evaluation of lifestyle risk factors and absenteeism after two years in a worksite health promotion program. Am J Health Promot. 1989;4:128-133.

25. Stein AD, Shakour SK, Zuidema RA. Financial incentives, participation in employer-sponsored health promotion, and changes in employee health and productivity: HealthPlus Health Quotient program. J Occup Environ Med. 2000;42: 1148-1155.

26. Bertera RL. The effects of workplace health promotion on absenteeism and employment costs in a large industrial population. Am J Public Health. 1990;80: 1101-1105.

27. Serxner S, Gold D, Anderson D, Williams D. The impact of a worksite health promotion program on short-term disability usage. J Occup Environ Med. 2001; 43:25-29.

28. Maniscalco P, Lane R, Welke M, Mitchell JH, Husting L. Decreased rate of back injuries through a wellness program for offshore petroleum employees. J Occup Environ Med. 1999;41:813-820.

29. The Health Project. C. Everett Koop National Health Award Winners. 1994
Winners. Available at: http://healthproject. stanford. edu/koop/1994. html. Accessed April 11, 2002.

30. Yen L, Edington DW, Witting P. Associations between health risk appraisal scores and employee medical claims costs in a manufacturing company. Am J Health Promot. 1991;6:46-54.

31. Yen L, Edington DW, Witting P. Prediction of prospective medical claims costs and absenteeism costs for 1284 hourly workers from a manufacturing company. J Occup Med. 1992;34:428-435.

32. Yen L, Edington DW, Witting P. Corporate medical claim cost distributions and factors associated with high-cost status. J Occup Med. 1994;36:505-515.

33. Edington DW, Yen L, Witting P. The financial impact of changes in personal health practices. J Occup Environ Med. 1997;39:1037-1046.

34. SAS Institute Inc. SAS/STAT User's Guide, Version 6, Fourth Edition, Volumes 1\&2, Cary, NC: SAS Institute Inc.; 1989.

35. Lipold AG. Managing the guy who isn't there. Bus \& Health. 2000;18:25-30.

36. Edington DW. Emerging research: a view from one research center. Am J Health Promot. 2001;15:341-349.

37. Bunn WB, Pikelny DB, Slavin TJ, Paralkar S. Health, safety, and productivity in a manufacturing environment. J Occup Environ Med. 2001;43:47-55.

38. Goetzel RZ, Guindon AM, Turshen IJ, Ozminkowski RJ. Health and productivity management: establishing key performance measures, benchmarks, and best practices. J Occup Environ Med. 2001; 43:10-17. 